\title{
Discarded weight and entanglement spectra in the Numerical Renormalization Group
}

\author{
A. Weichselbaum \\ Physics Department, Arnold Sommerfeld Center for Theoretical Physics, \\ and Center for NanoScience, Ludwig-Maximilians-Universität, 80333 Munich, Germany
}

(Dated: May 27, 2022)

\begin{abstract}
A quantitative criterion to prove and analyze convergence within the numerical renormalization group (NRG) is introduced. By tracing out a few further NRG shells, the resulting reduced density matrices carry relevant information on numerical accuracy as well as entanglement. Their spectra can be analyzed twofold. The smallest eigenvalues provide a sensitive estimate of how much weight is discarded in the low energy description of latter iterations. As such, the discarded weight indicates in a site-specific manner whether sufficiently many states have been kept in a single NRG run. The largest eigenvalues of the reduced density matrices, on the other hand, lend themselves to a straightforward analysis in terms of entanglement spectra, which can be combined into entanglement flow diagrams. The latter show strong similarities with the well-known standard energy flow diagram of the NRG, supporting the prevalent usage of entanglement spectra to characterize different physical regimes.
\end{abstract}

PACS numbers: $\quad$ 02.70.-c, 05.10.Cc, 75.20.Hr, 78.20.Bh

\section{INTRODUCTION}

The numerical renormalization group $(\mathrm{NRG})^{1}$ is a powerful method that provides a highly systematic nonperturbative approach to the wide realm of so-called quantum impurity systems. These consist of an arbitrary small quantum system (the impurity) in contact with a macroscopic non-interacting usually fermionic bath. Each part is simple to solve exactly on its own. In presence of interaction at the location of the impurity, however, the combination of both gives rise to strongly-correlated quantum-many-body phenomena. ${ }^{2}$ Wilson's logarithmic coarse-graining of the bath leads to a semi-infinite chain with exponentially decaying couplings, which justifies the concept of energy scale separation. That is the Wilson chain can be diagonalized iteratively by adding one site at a time and retaining the lowest $M_{\mathrm{K}}$ states only. The obvious question, however, is how many states should one keep on average for convergence in this procedure? At a given iteration there is no quantitative a priori measure that indicates how many low-energy states are required for a proper description of the remaining low-energy physics. Usually, the only way to check convergence within the NRG is by repeating the entire calculation and showing that the results no longer change when further increasing $M_{\mathrm{K}}$. Therefore an NRG calculation is typically run somewhat blindly for some pre-determined $M_{\mathrm{K}}$.

This somewhat uncontrolled truncation in the NRG is in stark contrast to the situation in the density matrix renormalization group (DMRG). ${ }^{3-5}$ DMRG is based on a (strictly) variational principle, and as such has a clean well-defined truncation of the state space for part of the system through the discarded weight in its reduced density matrix. ${ }^{4}$ In contrast to the less suggestive plain number $M_{\mathrm{K}}$ of states kept, the discarded weight represents a reliable quantitative measure for the accuracy of a calculation. Within the DMRG, $M_{\mathrm{K}}$ can be easily ad- justed according to some predefined threshold in the discarded weight, instead. Motivated by DMRG then, an approximate similar criterion can be established within the NRG as will be shown in the following. The analysis requires a slightly longer chain, as shown schematically in Fig. 1. With the extra $n_{0}$ sites traced out again from the ground state space of the enlarged system, this allows to estimate the discarded weight. The latter offers a quantitative convergence measure that is specifically of interest for numerically expensive models such as multichannel models, or models where the energy scale separation along the Wilson chain might be in question due to modifications in the discretized Hamiltonian. In either case, a small discarded weight provides a strong indication for converged NRG data.

Furthermore, the reduced density matrices generated for the evaluation of the discarded weight also allow a quite different analysis in terms of their dominant correlations. In particular, combining their entanglement spectra into entanglement flow diagrams, this offers a complementary view to the usual NRG energy flow diagram, which is entirely based on the analysis of the lowenergy state space of a prior NRG run.

The paper is thus organized as follows. In Sec. I the essentials of the numerical renormalization group are re-

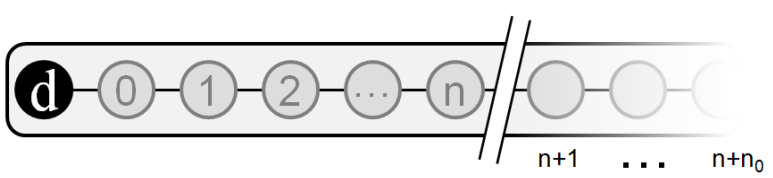

Figure 1: Schematic depiction of tracing out the low-energy sector of the Wilson chain at iteration $n$ by including and analyzing $n_{0}$ more NRG iterations. The impurity (dot) is entirely contained in the first site, while the bath is coarsegrained and mapped onto the remaining semi-infinite tightbinding chain of sites $n=0,1,2, \ldots$ 
visited, including the construction of reduced density matrices. Sec. II then uses a specific set of reduced density matrices in the definition and analysis of the discarded weight within the NRG. Sec. III offers a complementary view on these reduced density matrices by analyzing their entanglement content in terms of entanglement spectra. Sec. IV, finally, summarizes and includes an outlook.

\section{A. The numerical renormalization group}

Within the NRG, the continuum of the non-interacting bath of half-bandwidth $W$ is logarithmically coarsegrained in energy space, followed by an exact mapping onto a semi-infinite so-called Wilson-chain. ${ }^{1,6}$ The impurity space is coupled to the first site of this chain only, as depicted schematically in Fig. 1. The logarithmic coarse-graining is defined through the dimensionless discretization parameter $\Lambda>1$. With the chemical potential at energy zero, the continuum of states in the energy intervals $\pm W\left[\Lambda^{-(n-z+1)}, \Lambda^{-(n-z)}\right]$ is effectively represented by single fermionic levels (coarsegraining), including an arbitrary z-shift with $z \in\left[0,1\left[.^{7-9}\right.\right.$ The subsequent exact mapping onto the semi-infinite chain (Lanczos tridiagonalization) ${ }^{10}$ results in an effective tight-binding chain with the exponentially decaying hopping $t_{n} \sim \Lambda^{-n / 2}$ between sites $n$ and $n+1$. For sufficiently large $\Lambda$, typically $\Lambda \gtrsim 1.7$, this then justifies the essential NRG assumption of energy scale separation: by iterative diagonalization of the Wilson chain by adding one site at a time, large energies are considered first, with the (approximate) eigenstates at large energies discarded and considered unimportant in the description of the lower energy scales still to follow. Thus each site of the Wilson chain corresponds to an energy shell with a characteristic energy scale,

$$
\omega_{n} \equiv \frac{\Lambda^{z-1}(\Lambda-1)}{\log \Lambda} W \Lambda^{-\frac{n}{2}} .
$$

Here, the prefactor was chosen such, that the rescaled couplings $\lim _{n \rightarrow \infty}\left(t_{n} / \omega_{n}\right)=1$ quickly approach unity for longer Wilson chains for arbitrary $\Lambda$ and z-shift, with the discretization following the prescription of [9] for a flat hybridization, i.e. $\Gamma(E)=\Gamma \theta(W-|E|)$.

With $\hat{H}_{n}$ the full Hamiltonian $\hat{H}$ of the Wilson chain up to and including site $n$, its low-energy eigenstates are given by the NRG eigenstates $\hat{H}_{n}\left|s_{n}\right\rangle=E_{s}^{n}\left|s_{n}\right\rangle$. Complemented by an arbitrary state $\left|e_{n}\right\rangle$ for the remainder of the system following site $n$, the NRG assumption of energy scale separation can be summarized then in the following approximation, ${ }^{11}$

$$
\hat{H}|s e\rangle_{n} \simeq E_{s}^{n}|s e\rangle_{n},
$$

i.e. the states $|s e\rangle_{n} \equiv\left|s_{n}\right\rangle \otimes\left|e_{n}\right\rangle$ are, to a good approximation, also eigenstates of the entire Wilson chain. The energies $E_{s}^{n}$ at iteration $n$ are usually expressed relative to the ground state energy of that iteration, and rescaled by a factor $\frac{W}{2}(\Lambda+1) \Lambda^{-n / 2} \propto \omega_{n}$ to resolve the energy shell at iteration $n$. The resulting energies are referred to as rescaled energies. For fully fermionic systems, they typically show an intrinsic even-odd behavior. Thus combining the rescaled energies vs. even and odd iterations $n$ separately, this results in the standard energy flow diagrams of the NRG. ${ }^{1,6}$

The approximate many-body eigenstates $|s e\rangle_{n}$ are constructed iteratively, and therefore described in terms of matrix-product-states. ${ }^{5,12-14}$ Each iterative step results in a basis transformation, encoded in an A-tensor, that combines an existing effective basis $\left|s_{n}\right\rangle$ for the system up to and including site $n$ with the state space $|\sigma\rangle$ of site $n+1$,

$$
\left|s_{n+1}\right\rangle=\sum_{s_{n}^{\prime}, \sigma_{n+1}}\left|s_{n}^{\prime}, \sigma_{n+1}\right\rangle \underbrace{\left\langle s_{n}^{\prime}, \sigma_{n+1} \mid s_{n+1}\right\rangle}_{\equiv A_{s_{n}^{\prime} s_{n+1}}^{\left[\sigma_{n+1}\right]}},
$$

with $\left|s_{n}^{\prime}, \sigma_{n+1}\right\rangle \equiv\left|s_{n}^{\prime}\right\rangle \otimes\left|\sigma_{n+1}\right\rangle$. The orthogonality of state spaces, $\left\langle s_{n+1} \mid s_{n+1}^{\prime}\right\rangle=\delta_{s s^{\prime}}$, directly implies the orthonormality relation for $A$-tensors, ${ }^{4}$

$$
\sum_{\sigma_{n+1}} A^{\left[\sigma_{n+1}\right] \dagger} A^{\left[\sigma_{n+1}\right]}=\mathbf{1} .
$$

Without truncation, the dimension $M_{n}$ of the state space $\left|s_{n}\right\rangle$ increases exponentially with the number of sites included, $M_{n} \sim d^{n}$, with $d$ the dimension of a local Wilson site. Therefore the maximum number of states $M_{\mathrm{K}}$, that one can maintain in a calculation, is quickly reached after $n_{0} \simeq \log \left(M_{\mathrm{K}}\right) / \log (d)$ iterations. For every subsequent iteration, the state space $\left|s_{n}\right\rangle$ is truncated by retaining the lowest $M_{\mathrm{K}}$ states in energy only. This leads to the distinction between $\left|s_{n}^{\mathrm{K}}\right\rangle$ and $\left|s_{n}^{\mathrm{D}}\right\rangle$ for kept and discarded states at iteration $n$, respectively. Correspondingly, this also splits the A-tensor into two parts, $A_{K K}$ and $A_{K D}$, that propagate the state kept space from the previous iteration into the newly generated kept or discarded space, respectively.

The truncation criteria with respect to a fixed prespecified $M_{\mathrm{K}}$ can be softened towards a energy cutoff, ${ }^{2}$ $E_{\mathrm{K}}$, that is taken constant in rescaled energies. For a fair comparison for different z-shifts, it will be specified in units of the energy scale $\omega_{n}$ in Eq. (1). Since NRG data typically appears bunched at certain energies (e.g. see Fig. 3 later), $E_{\mathrm{K}}$ may hit a "gap" in the NRG spectrum at some iteration, and the last "bunch" of states included may lie, on average, at clearly smaller energies than $E_{\mathrm{K}}$. Given the empirical importance of the first few NRG iterations, therefore as a safety measure, by default, $E_{\mathrm{K}}$ was taken by $20 \%$ larger for the very first iteration where truncation occurred, i.e. using $1.2 E_{\mathrm{K}}$ there with $E_{\mathrm{K}}$ specified in context. Typical values are in the range $E_{\mathrm{K}}=5 \ldots 8$.

The model system considered in this paper is the well-known standard single impurity Anderson model 
(SIAM),

$$
\begin{aligned}
H_{N}^{\mathrm{SIAM}} & =\sum_{\sigma} \varepsilon_{d \sigma} \hat{n}_{\sigma}+U \hat{n}_{d \uparrow} \hat{n}_{d \downarrow}+\sum_{\sigma} \sqrt{\frac{2 \Gamma}{\pi}}\left(\hat{d}_{\sigma}^{\dagger} \hat{f}_{0 \sigma}+\text { h.c. }\right) \\
& +\sum_{\sigma} \sum_{n=0}^{N-1} t_{n}\left(\hat{f}_{n, \sigma}^{\dagger} \hat{f}_{n+1, \sigma}+\text { h.c. }\right)
\end{aligned}
$$

with the operators $\hat{d}_{\sigma}^{\dagger}\left(\hat{f}_{n \sigma}^{\dagger}\right)$ creating a particle with spin $\sigma \in\{\uparrow, \downarrow\}$ at the impurity (at site $n$ in the bath), respectively, having $\hat{n}_{d \sigma} \equiv \hat{d}_{\sigma}^{\dagger} \hat{d}_{\sigma}$. The energy $\varepsilon_{d \sigma} \equiv$ $\varepsilon_{d}-\frac{B}{2}\left(\hat{n}_{d \uparrow}-\hat{n}_{d \downarrow}\right)$ is the spin dependent level-position of the impurity in the presence of a magnetic field $B$. Furthermore, $U$ is the onsite Coulomb interaction and $\Gamma$ the hybridization of the impurity with the bath. All parameters will be specified in units of the bandwidth $W:=1$ in context with the figure panels. The bath in Eq. (5) is already represented in terms of a Wilson chain, ${ }^{1}$ described by the semi-infinite tight binding chain $(N \rightarrow \infty)$ with exponentially decaying hopping amplitudes $t_{n} \sim \Lambda^{-n / 2}$. In practice, $N$ can be taken finite, with $\hat{H}_{n}$ describing the Wilson chain up to and including site $n \leq N$.

Charge and spin are conserved in the SIAM in Eq. (5), where, however, only the abelian part of the symmetries is included in the calculations. Hence the number of states $M_{\mathrm{K}}$ directly refers to the actual number of states kept in a calculation (in contrast to the dimension of reduced multiplet spaces with non-abelian symmetries). Similarly, also the discussion of the entanglement spectra further below will refer to the abelian symmetry labels which also applies when non-abelian symmetries are broken. Note that while, in general, a particle-hole symmetric impurity setting will be used, this can be easily broken by applying a (small) gating potential to the impurity level. Moreover, the SU(2) spin symmetry, in fact, will be broken explicitly by the application of an external magnetic field.

\section{B. Density matrices}

The NRG eigenbasis of Eq. (2) with respect to the discarded space forms a complete many-body eigenbasis. ${ }^{11}$ Initially introduced for the feat of real time-evolution within the NRG, this eigenbasis is actually applicable and tractable more generally within the NRG framework. ${ }^{15}$ In particular, this allows the clean calculation of correlation functions in terms of the full density matrix (FDM) in the many-body eigenbasis, ${ }^{12}$ in that

$$
\hat{\rho}(T) \equiv \frac{1}{Z} e^{-\beta \hat{H}} \cong \frac{1}{Z} \sum_{n s e} e^{-\beta E_{s}^{n}}|s e\rangle_{n n}^{\mathrm{DD}}\langle s e|,
$$

with $\beta \equiv 1 / k_{B} T$ for arbitrary temperatures $T$, using non-rescaled energies $E_{s}^{n}$ relative to a common energy reference, by construction of a thermal density matrix. Eq. (6) can be rewritten as $\hat{\rho}(T) \equiv \sum_{n} w_{n}(T) \hat{\rho}_{n}(T)$, i.e. a normalized distribution $\sum_{n} w_{n}=1$ of the density matrices $\hat{\rho}_{n}(T)$ generated in the basis of iteration $n .{ }^{12}$ For

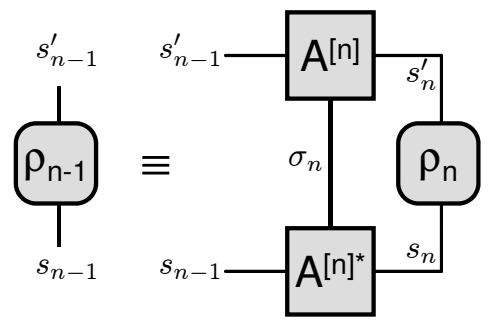

Figure 2: Backward update of given density matrix $\rho_{n}$ at iteration $n$. Blocks represent data spaces, lines correspond to indices. The lines connecting different blocks are contracted indices (i.e. indices summed over), such as $\sigma_{n}, s_{n}$ and $s_{n}^{\prime}$, while open lines represent open indices, e.g. the indices $s_{n-1}$ and $s_{n-1}^{\prime}$.

a given temperature $T$, the distribution $w_{n}$ is strongly peaked around iteration $n_{\mathrm{T}}$ that corresponds to the energy scale of temperature. Hence temperature essentially terminates the Wilson chain.

In this paper, however, mainly reduced density matrices derived from ground states will be considered, hence temperature is essentially zero. More generally then, consider an arbitrary density matrix defined in the manybody basis $\left|s_{n}\right\rangle$ of iteration $n$ in either kept or discarded space, $\mathrm{X} \in\{\mathrm{K}, \mathrm{D}\}$,

$$
\hat{\rho}_{n}^{[\mathrm{X}]} \equiv \sum_{s_{n} s_{n}^{\prime} \in \mathrm{X}} \rho_{s_{n} s_{n}^{\prime}}^{[\mathrm{X}]}\left|s_{n}\right\rangle\left\langle s_{n}^{\prime}\right|
$$

where $\rho_{n}^{[\mathrm{X}]}$ (i.e. without the hat) represents the space of matrix elements $\rho_{s_{n} s_{n}^{\prime}}^{[\mathrm{X}]}$. The prototypical and well-known operation on such a density matrix is tracing out the last site $n,{ }^{11,12,15-17}$

$$
\begin{aligned}
\hat{\rho}_{n-1}^{[\mathrm{K}]} & =\sum_{\substack{s_{n-1}, s_{n-1}^{\prime} \\
\sigma_{n}}}\left(A_{K X}^{\left[\sigma_{n}\right]} \rho_{n}^{[\mathrm{X}]} A_{K X}^{\left[\sigma_{n}\right] \dagger}\right)_{s_{n-1} s_{n-1}^{\prime}}\left|s_{n-1}\right\rangle\left\langle s_{n-1}^{\prime}\right| \\
& \equiv \hat{\mathcal{P}}_{n} \hat{\rho}_{n}^{[\mathrm{X}]}
\end{aligned}
$$

written as a matrix product of the matrices $A_{K X}^{\left[\sigma_{n}\right](\dagger)}$ and $\rho_{n}^{[\mathrm{X}]}$ in the first line. Equation (8), in the following referred to as backward update, introduces the notational shorthand $\hat{\mathcal{P}}_{n}$ for the bilinear product of the $A$ - and $A^{*}$ tensor at site $n$, that acts as a linear superoperator on the density matrix $\hat{\rho}_{n}$. The corresponding contraction pattern is shown in a simple graphical depiction in Fig. 2. By construction, the backward update of a density matrix in Eq. (8) always results in a density matrix in the kept space of the earlier iteration, and with Eq. (4) representing a complete positive map, Eq. (8) clearly also preserves the properties of a density matrix. 


\section{DISCARDED WEIGHT WITHIN THE NRG}

The standard notion of NRG is that it zooms in towards the low energy sector of a given many-body Hamiltonian, while iteratively discarding states at higher energies. Having a semi-infinite chain, this can continue to arbitrarily small energy scales, which enables NRG to resolve dynamically generated small energy scales as they appear, for example, in the context of Kondo physics. From a variational point of view for matrix-productstates, this implies that the cost function can be identified as

$$
\lim _{N \rightarrow \infty}\left\langle s_{N}\left|H_{N}\right| s_{N}\right\rangle \rightarrow \mathrm{MIN}
$$

yielding the ground state $|0\rangle_{\infty}$ of the semi-infinite Wilson chain. For a sufficiently long chain of total length $N$ then included in a given calculation, the state $|0\rangle_{N}$ will be referred to as the overall ground state of this Wilson chain. In fact, the cost function in Eq. (9) is well captured within the NRG through its principle of energy scale separation. ${ }^{18}$

If at a given iteration within the NRG states essentially decouple with respect to the low energy state space still to follow, these states will quickly and efficiently be discarded as high energy states. The truncation towards the low-energy sector also implies, that the state space at large energies is necessarily more crudely resolved, consistent with the coarser discretization there. The lowest $M_{\mathrm{K}}$ states kept at a given iteration $n$ then are important for the correct description of the low-energy sector still to come. However, there is no real quantitative a-priori measure to indicate whether the number $M_{\mathrm{K}}$ of states to be kept is appropriate. Conversely, however, at a given iteration $n$ one can ask whether all states kept a few iterations earlier were actually important. This question can be answered entirely within the kept spaces of these iterations, hence is numerically cheap to analyze.

\section{A. Construction of reduced density matrices}

Consider the actual ground state space $G$ at some arbitrary but fixed iteration $n^{\prime}$. In general, it may be $g_{n^{\prime}}$-fold degenerate, hence consider its fully mixed density matrix,

$$
\hat{\rho}_{0, n^{\prime}} \equiv \frac{1}{g_{n^{\prime}}} \sum_{s \in G}\left|s_{n^{\prime}}\right\rangle\left\langle s_{n^{\prime}}\right| .
$$

By construction, the number of eigenvalues of $\hat{\rho}_{0, n^{\prime}}$ unequal zero, i.e. its Schmidt rank, is equal to $g_{n^{\prime}}$. Now, tracing out the last iteration $n^{\prime}$, i.e. the lowest energy scale included in $\hat{\rho}_{0, n^{\prime}}$, is equivalent to the backpropagation $\hat{\rho}_{0}^{\left[n^{\prime}-1 ; 1\right]} \equiv \hat{\mathcal{P}}_{n^{\prime}} \hat{\rho}_{0, n^{\prime}}$ in Eq. (8). Through this operation, the Schmidt rank will rise, in general, by a factor of $d$, with $d$ the state space dimension of a Wilson site. Repeating this process iteratively, this allows to trace out the $n_{0}$ smallest energy shells in $\hat{\rho}_{0, n^{\prime}}$. Thus with $n^{\prime}=n+n_{0}$, this leads to the reduced density matrix,

$$
\begin{aligned}
\hat{\rho}_{0}^{\left[n ; n_{0}\right]} & \equiv\left(\prod_{l=n+1}^{n+n_{0}} \hat{\mathcal{P}}_{l}\right) \hat{\rho}_{0, n+n_{0}} \\
& \equiv \sum_{s s^{\prime}}^{M_{\mathrm{K}}} \rho_{s s^{\prime}}^{\left[n ; n_{0}\right]}\left|s_{n}^{\mathrm{K}}\right\rangle\left\langle s_{n}^{\prime \mathrm{K}}\right|,
\end{aligned}
$$

which, by construction, is defined in the kept space of iteration $n$. The Schmidt rank will grow quickly, i.e. exponentially, in this process, until after $n_{0}$ iterations, with

$$
n_{0} \gtrsim \operatorname{ceil}\left[\log \left(M_{\mathrm{K}}\right) / \log (d)\right] \quad\left(n_{0} \ll N\right),
$$

it reaches the full dimension $M_{\mathrm{K}}$ of the kept space. Typically, $n_{0}$ is much smaller compared to the full length $N$ of the Wilson chain considered, and conversely also specifies the initial number of NRG iterations in a forward direction that can be typically performed without truncation. For the definition of the discarded weight below, it is sufficient to stop the back-propagation of $\hat{\rho}_{0, n+n_{0}}$ at this point.

The reduced density matrix $\hat{\rho}_{0}^{\left[n ; n_{0}\right]}$ generated in Eq. (11) is, in general, not diagonal in the energy eigenbasis $\left|s_{n}^{\mathrm{K}}\right\rangle$, since through the traced out lower-energy sites it does know about an enlarged system. Its eigenvectors are described by a unitary transformation $u_{r s^{\prime}}^{\left[n ; n_{0}\right]}$ within the NRG eigenstates kept at iteration $n$,

$$
\begin{gathered}
\left|r_{n ; n_{0}}\right\rangle \equiv \sum_{s^{\prime}} u_{r s^{\prime}}^{\left[n ; n_{0}\right]}\left|s_{n}^{\prime \mathrm{K}}\right\rangle, \\
\text { with } \quad \hat{\rho}_{0}^{\left[n ; n_{0}\right]}\left|r_{n ; n_{0}}\right\rangle=\rho_{r}^{\left[n ; n_{0}\right]}\left|r_{n ; n_{0}}\right\rangle,
\end{gathered}
$$

where the index $r$ shall refer to the eigenstates of the reduced density matrix, in contrast to the index $s$ for the energy eigenstates. Here, the eigenvalue $\rho_{r}^{\left[n ; n_{0}\right]}$ describes the importance of a specific linear superposition of NRG eigenstates at iteration $n$ for the low-energy description of latter iterations.

This offers two routes for the analysis of the density matrices $\hat{\rho}_{0}^{\left[n ; n_{0}\right]}$. (i) Adhering to the energy eigenbasis of the NRG, the importance of the kept state $\left|s_{n}^{\mathrm{K}}\right\rangle$ at eigenenergy $E_{s}^{n}$ for the latter low-energy physics is given by the expectation value

$$
\rho_{s}^{\left[n ; n_{0}\right]} \equiv\left\langle s_{n}^{\mathrm{K}}\left|\hat{\rho}_{0}^{\left[n ; n_{0}\right]}\right| s_{n}^{\mathrm{K}}\right\rangle,
$$

i.e. the diagonal matrix elements $\rho_{s s}^{\left[n ; n_{0}\right]}$. Alternatively, (ii) using the eigenbasis of the reduced density matrices, the weights of these states are given by the eigenvalues $\rho_{r}^{\left[n ; n_{0}\right]}$, while now their energies are given by the expectation values

$$
E_{r}^{\left[n ; n_{0}\right]} \equiv\left\langle r_{n ; n_{0}}\left|\hat{H}_{n}\right| r_{n ; n_{0}}\right\rangle
$$

Both routes will be analyzed and compared in the following. However, the actual eigendecomposition of the 


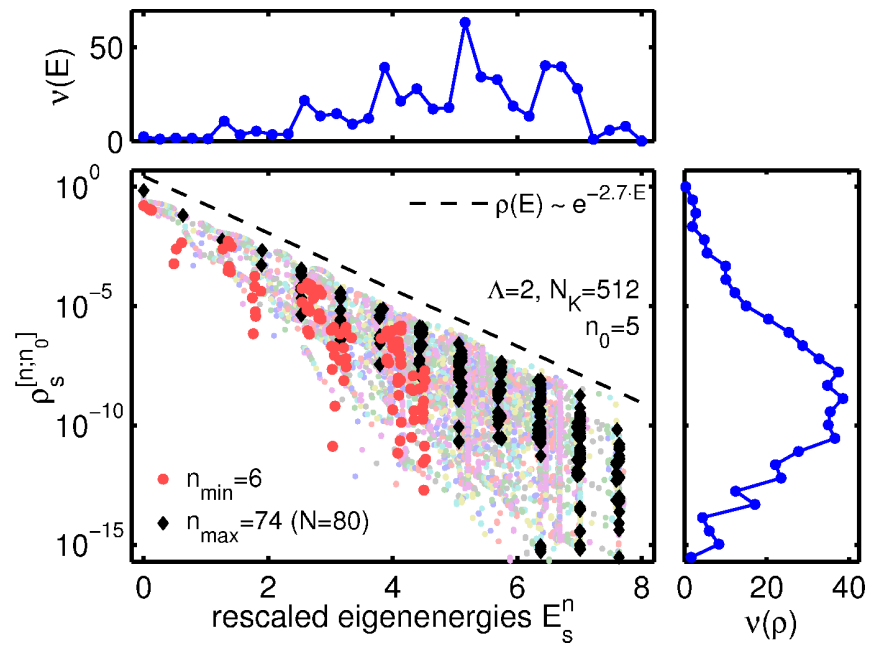

Figure 3: (Color online) Weight distribution of energy eigenstates over full NRG run at fixed $M_{\mathrm{K}}=512$ for the SIAM [Eq. (5): $\left.U=0.20, \epsilon_{d}=-U / 2, \Gamma=0.01\right]$. The main panel shows the rescaled eigenenergies $E_{s}^{n}$ vs. their weights $\rho_{s}^{\left[n ; n_{0}\right]}$ as in Eq. (14). Data is shown only for those iterations where truncation occurred, with data from the same iteration shown in the same color. The two iterations with smallest (largest) energy range, $n_{\max }\left(n_{\min }\right)$, are highlighted in strong colors (black diamonds (red crosses)), respectively, while light colors are used for all other iterations. The top [right] panel shows the energy [weight] distribution $\nu(E)[\nu(\rho)]$, Eq. (16) [Eq. (17)], respectively, for the data in the main panel, with matching energy $E$ [weight $\rho$ ] axis. The binning referred to in the text to Eqs. (16) and (17) is indicated by the intervals between the data points in the top and right panel.

reduced density matrices will be preferred for the remainder of the paper as explained.

In either case, a set of states $i$ with (average) energy $E_{i}$ is given together with their respective (average) weight $\rho_{i}$ that represents the states importance for latter iterations. For the first [second] route above this data is given by $\left(E_{s}^{n}, \rho_{s}^{\left[n ; n_{0}\right]}\right)\left[\left(E_{r}^{\left[n ; n_{0}\right]}, \rho_{r}^{\left[n ; n_{0}\right]}\right)\right]$, respectively. Given that the reduced density matrix $\hat{\rho}_{0}^{\left[n ; n_{0}\right]}$, by construction, exists in the kept space only, therefore also all states $i$ refer to the kept space or a linear superpositions thereof. Moreover, for every iteration, the weights $\rho_{i}$ are normalized, i.e. they are positive and add up to 1 , while by combining data from different iterations, the energies $E_{i}$ are always specified in rescaled units.

The resulting data $\left(E_{i}, \rho_{i}\right)$ then is clearly correlated. It is analyzed threefold, (i) in terms of the average distribution of the rescaled energies $E_{i}$

$$
\nu(E) \cong \frac{1}{N^{\prime}} \sum_{n=1}^{N} \sum_{E<E_{i}<E+d E} 1
$$

(ii) the average distribution of the weights $\rho_{i}$,

$$
\nu(\rho) \cong \frac{1}{N^{\prime}} \sum_{n=1}^{N} \sum_{\rho<\rho_{i}<\rho+d \rho} 1,
$$

and (iii) their average dependence on each other

$$
\begin{aligned}
\rho(E) & \cong \frac{1}{N^{\prime} d E} \sum_{n}^{N} \sum_{E<E_{i}<E+d E} \rho_{i} \\
& \cong \kappa e^{-\kappa E} .
\end{aligned}
$$

Here some appropriate linear (logarithmic) binning of the data is assumed with energy (weight) intervals $d E$ $(d \rho)$, respectively. In particular, the densities in Eqs. (16) and (17) are clearly dependent on these binning intervals, which therefore will properly indicated in the subsequent plots. The prime in the summation and the normalization indicates that only those iterations $n$ are included where state space truncation occurred, i.e. typically $n \gtrsim n_{0}$. The total number of these iterations is given by $N^{\prime}$. With chosen normalization then, the sum over the binned $\nu(E)$ and $\nu(\rho)$ data both yield the average number of kept states, while the integrated weight distribution $\rho(E)$ in Eq. (18a) is normalized to 1, since $\operatorname{tr}(\rho) \sim \int_{0}^{\infty} \rho(E) d E=1$. As will be seen later, the weight distribution $\rho(E)$ typically shows a clear exponential decay with a characteristic exponent $\kappa$, as indicated already in Eq. (18b), with the prefactor chosen such that it also preserves normalization.

\section{Energy eigenbasis}

The correlation between the eigenenergies $E_{s}^{n}$ and their corresponding weights $\rho_{s}^{\left[n ; n_{0}\right]}$ is plotted as a scatter plot in the main panel of Fig. 3. The model analyzed is the SIAM in Eq. (5) in the Kondo regime using a fixed number of kept states, with all parameters specified in the figure caption. The weights $\rho_{s}^{\left[n ; n_{0}\right]}$ clearly diminish exponentially with energy, which is intuitively expected as a consequence of energy scale separation within the NRG. The integrated weight distribution $\rho(E)$ (dashed black line, $c f$. Eq. 18a), shows a clear exponential decay with an exponent $\kappa \simeq 2.7$. As seen in Fig. 3, this distribution clearly also serves as an upper bound of the weights $\rho_{s}^{\left[n ; n_{0}\right]}$ at a given energy.

The upper panel in Fig. 3 shows the distribution $\nu(E)$ in Eq. (16) of the energies $E_{s}^{n}$ plotted in the main panel (matching horizontal axis). This distribution shows a strong increase with energy $E$, consistent with the notion that the many-body phase space grows quickly as the available energy for excitations becomes larger. Towards large energies, eventually, the data is necessarily truncated to the finite number $M_{\mathrm{K}}$ of kept states, which leads to a drop in the density $\nu(E)$. The exact boundary with respect to energy is somewhat blurred, though, 
since in given case fixed $M_{\mathrm{K}}$ allows the energy range to vary for different iterations $n$. The right panel of Fig. 3, on the other hand, shows the distribution $\nu(\rho)$ in Eq. (17) of the weights $\rho_{s}^{\left[n ; n_{0}\right]}$ plotted in the main panel (matching vertical axis). This distribution is peaked around the largest weights $\rho_{s}^{\left[n ; n_{0}\right]}$ for the largest energies $E_{s}^{n}$.

The data in the main panel of Fig. 3 is typically bunched around a set of energies for a fixed iteration $n$. This is also reflected in the distribution $\nu(E)$ in the upper panel of Fig. 3, and is due to the discretization of the model. Moreover, two iterations are highlighted in strong colors. These correspond to the iterations whose energy range is smallest $\left(n_{\min }=6\right.$, red bullets) or largest $\left(n_{\max }=74\right.$, black diamonds). Intuitively, the largest numerical error is expected from iterations such as $n_{\text {min }}$ (red bullets) since through Eq. (18b), stopping at premature energies directly translates to largest missing, i.e. discarded weight in the density matrix. As an aside, this serves as a strong argument in favor of truncation w.r.t. a fixed energy cutoff $E_{\mathrm{K}}$ rather than a fixed number $M_{\mathrm{K}}$ of states. Fixed $E_{\mathrm{K}}$, however, also introduces more noise to the data in particular for higher lying states. Hence both truncations will be used and pointed out in context.

The weights $\rho_{s}^{\left[n ; n_{0}\right]}$ in the main panel of Fig. 3 show significant vertical spread, which translates into a pronounced tail towards exponentially smaller $\rho$ in the distribution $\nu(\rho)$ in the right panel. For a given energy $E$ therefore, many of the states have order of magnitudes lower weight than the top-most weights close to $\rho(E)$ in the main panel. This indicates that the energy representation with its corresponding diagonal weights $\rho_{s}^{\left[n ; n_{0}\right]}$ is not necessarily the optimal basis to analyze accuracy. Moreover, note that using the energy eigenbasis $\left|s_{n}\right\rangle$ with energies $E_{s}^{n}$ in the analysis of the reduced density matrices, this actually mingles the energy scales of an effectively larger system $\hat{H}_{n+n_{0}}$ with the basis generated w.r.t. $\hat{H}_{n}$ only.

\section{Eigenbasis of reduced density matrices}

From the point of view of a variationally optimal representation of the ground state space of an enlarged system, on the other hand, one is directly led to the eigenspectrum of the reduced density matrix, as exemplified within DMRG. ${ }^{3}$ The analysis of Fig. 3 therefore is repeated for the same underlying Wilson chain, yet with two modifications: (i) the eigendecomposition of the reduced density matrices in Eq. (13) together with Eq. (15) is used instead of the energy eigenbasis, and furthermore (ii) the NRG truncation criterion is based on a fixed energy cutoff, $E_{\mathrm{K}}=6$. The results are shown in Fig. 4, with striking quantitative differences compared to Fig. 3. The spread in the scatter plot is significantly narrowed, and overall, the data decays much faster with $\kappa \simeq 4.6$, cf. Eq. (18b). Therefore this leads to a clearly improved separation of the actually relevant states for the subsequent description

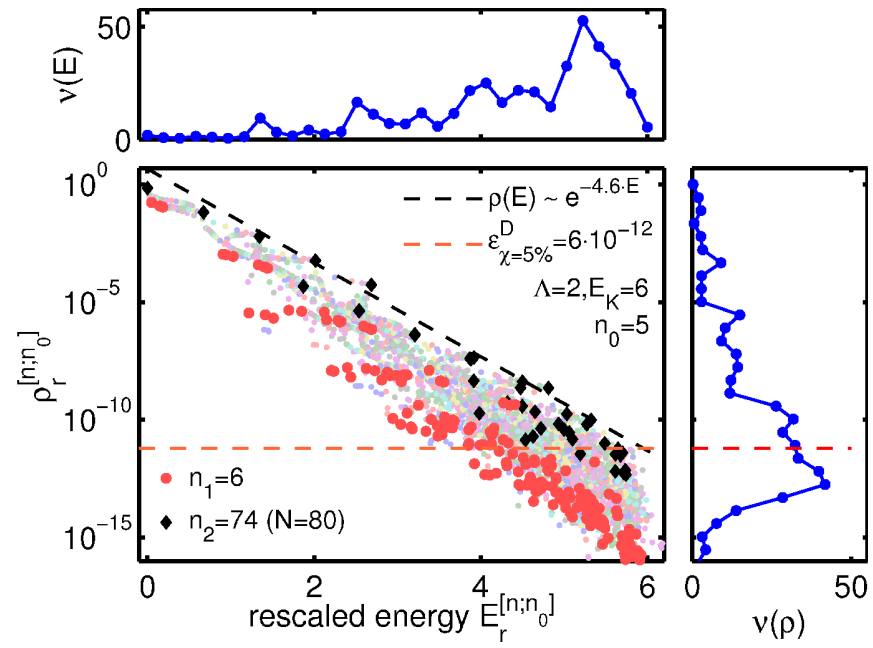

Figure 4: (Color online) Similar analysis as in Fig. 3 (see caption there for further information) for the same underlying Hamiltonian, except that the eigenspectrum of the reduced density matrices in Eq. (13) was used together with Eq. (15) and a fixed energy cutoff $E_{\mathrm{K}}=6$. Similar to Fig. 3, only those iterations are shown where truncation occurred (same color for data from the same iteration), with the same two iterations highlighted as in Fig. 3, indicated by $n_{1}$ and $n_{2}$. The estimate for the overall discarded weight $\varepsilon_{\chi=5 \%}^{\mathrm{D}} \simeq 6 \cdot 10^{-12}$ as defined in Eq. (21) is indicated by the horizontal dashed line.

of the lower-energy scales. This suggests that many of the NRG eigenstates, as their energy increases, loose importance much faster as compared to Fig. 3, despite the relatively large diagonal weights $\rho_{s}$ in the density matrix still seen there. In a sense, the weights there represent mere matrix-elements in a non-diagonal representation.

The iterations highlighted in Fig. 4 are the same iterations as in Fig. 3. Given a fixed energy cutoff $E_{\mathrm{K}}=6$ here, however, both have a comparable energy range (hence the altered notation $n_{1}$ and $n_{2}$ ), with the number $M_{\mathrm{K}}$ of kept states varying from $\sim 1000$ at very early iterations (in particular iteration $n_{1}$ ), down to $\sim 250$ at late iterations (such as iteration $n_{2}$ ). Note also the markedly fewer data points seen for iteration $n_{2}$. This is only partly due to the reduced number of states, as there are also large systematic (approximate) degeneracies at the strong-coupling Kondo fixed point already reached at this iteration. This results in many of the black diamonds lying indistinguishably on top of each other (see also discussion on entanglement spectra later).

As seen from above discussion, rather than taking the energy eigenstates $\left|s_{n}\right\rangle$ and the corresponding diagonal matrix elements $\rho_{s}^{\left[n ; n_{0}\right]}$ (Fig. 3), the eigenvalues $\rho_{r}^{\left[n ; n_{0}\right]}$ of the reduced density matrix $\hat{\rho}_{0}^{\left[n ; n_{0}\right]}$ do represent a clearly better choice for the analysis of accuracy or entanglement in the system (Fig. 4), and thus will be used henceforth. This prescription also shows a more systematic exponential decay all the way down to numerical double precision noise $\left(10^{-16}\right)$, with the decay rate $\kappa$ of $\rho(E)$ roughly in- 


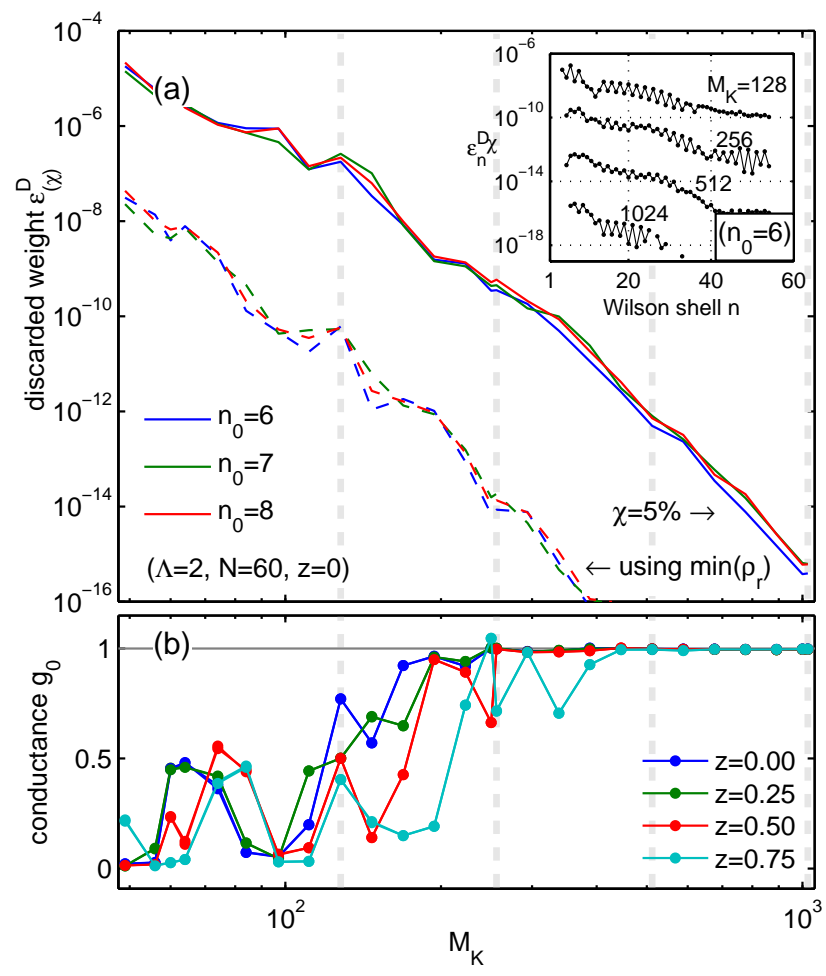

Figure 5: (Color online) Discarded weight $\varepsilon^{\mathrm{D}}$ for the SIAM [Eq. (5): $U=0.20, \epsilon_{d}=-U / 2, \Gamma=0.01$ (same parameters as in Fig. 3), with $\left.T_{K} \simeq 1.23 \cdot 10^{-5}\right]$. Panel (a) shows the discarded weight $\varepsilon_{(\chi)}^{\mathrm{D}}$ defined in Eq. (21) vs. $M_{\mathrm{K}}$ using $n_{0} \in\{6,7,8\}$. The data $\varepsilon_{\chi=5 \%}^{\mathrm{D}}$ is shown in solid lines, while the data based on the minimum eigenvalue of $\hat{\rho}_{0}^{\left[n ; n_{0}\right]}$ (cf. Eq. 19b) is shown in dashed lines. The distribution of the discarded weight $\varepsilon_{n}^{\mathrm{D}_{\chi}}$ along the Wilson chain is shown in the inset for $M_{\mathrm{K}} \in\{128,256,512,1024\}$, also marked by the vertical dashed lines in the main panels. Panel (b) shows the conductance $g_{0}$ vs. $M_{\mathrm{K}}$ in units of $2 e^{2} / h$ while using a set of shifted discretizations, with the z-values as specified. Convergence in the conductance towards the expected unitary limit is seen for $M_{\mathrm{K}} \gtrsim 400$, i.e. $\varepsilon_{\chi}^{\mathrm{D}} \lesssim 10^{-12}$.

dependent of the discretization parameter $\Lambda$.

\section{Definition of discarded weight}

With the motivation above, the definition of the discarded weight is based on the eigendecomposition of the reduced density matrices $\hat{\rho}_{0}^{\left[n ; n_{0}\right]}$ in Eq. (11), using the combined data of Eq. (13) and Eq. (15). In terms of Fig. 4, adding more states to the calculation essentially extends the data to larger energies and smaller weights, while the large-weight low-energy sector already remains widely intact. Therefore the largest discarded weight, i.e. the weight missing by states not included and hence not available, can be estimated, to a good approximation, up to an overall prefactor by the smallest weights in the kept state space, which are easily accessible. Given the expo- nential decay of the weights together with the residual spread in the data as seen in Fig. 4, the discarded weight at given iteration $n$ can thus be defined through the average weights $\rho_{r}^{\left[n ; n_{0}\right]}$ for the highest energies $E_{r}^{\left[n ; n_{0}\right]}$ in the kept space,

$$
\varepsilon_{n ; n_{0}}^{\mathrm{K}_{\chi}} \equiv\left\langle\rho_{r}^{\left[n ; n_{0}\right]}\right\rangle_{E_{r}^{\left[n ; n_{0}\right]} \geq(1-\chi) \max \left(E_{r}^{\left[n ; n_{0}\right]}\right)} .
$$

The parameter $\chi \ll 1$ is considered small, yet is chosen large enough (typically $\chi \simeq 0.05$ ) to average over the residual spread of weights. Alternatively and for comparison, an even simpler measure in terms of the minimum eigenvalue of $\hat{\rho}_{0}^{\left[n ; n_{0}\right]}$ will be considered,

$$
\varepsilon_{n ; n_{0}}^{\mathrm{K}} \equiv \min \left(\rho_{r}^{\left[n ; n_{0}\right]}\right),
$$

which no longer makes any explicit reference to energies. Note that even though $\varepsilon_{n ; n_{0}}^{\mathrm{K}_{\chi}}$ or $\varepsilon_{n ; n_{0}}^{\mathrm{K}}$, written $\varepsilon_{n ; n_{0}}^{\mathrm{K}_{(\chi)}}$ in short, are purely determined within the kept space, they clearly represent a sensible estimate for the discarded weight at iteration $n$, i.e. $\varepsilon_{n}^{\mathrm{D}_{(\chi)}} \sim \varepsilon_{n ; n_{0}}^{\mathrm{K}}$, defined as the fraction of relevant state space missing from the latter description of the low energy physics. If no truncation has occurred at iteration $n$, however, such as typically for the first $n<n_{0}$ iterations, of course, then there is no truncation error either, hence $\varepsilon_{n}^{\mathrm{D}_{(\chi)}}=0$ for these iterations.

In summary, the discarded weight $\varepsilon_{n}^{\mathrm{D}}$ at iteration $n$ is defined as follows,

$$
\varepsilon_{n}^{\mathrm{D}_{(\chi)}} \equiv \begin{cases}\varepsilon_{n ; n_{0}}^{\mathrm{K}_{(\chi)}} & \text { in the presence of truncation } \\ 0 & \text { without truncation at iteration } n\end{cases}
$$

Here $\frac{K_{n ; n_{0}}}{\varepsilon_{n}}$ can be determined efficiently by including and analyzing $n_{0}$ further NRG iterations within the kept space, where typically $n_{0} \ll N$, cf. Eq. (12). The overall discarded weight $\varepsilon_{(\chi)}^{\mathrm{D}}$ of a full NRG run then is taken, for simplicity, as the largest discarded weight per iteration,

$$
\varepsilon_{(\chi)}^{\mathrm{D}} \equiv \max _{n}\left(\varepsilon_{n}^{\mathrm{D}_{(\chi)}}\right)
$$

Using $\chi=5 \%$ as in Eq. (19a), the discarded weight for the NRG run in Fig. 4 is estimated by $\varepsilon_{\chi}^{\mathrm{D}} \simeq 6 \cdot 10^{-12}$, indicated by the horizontal dashed line. As seen from Fig. 4, the overall discarded weight $\varepsilon_{\chi}^{\mathrm{D}}$ for an NRG run essentially coincides with $\rho(E)$ at the largest energies within the kept space. On the other hand, $\varepsilon^{\mathrm{D}}$, i.e. without the usage of $\chi$ based on the plain minimum eigenvalue of the reduced density matrices $\hat{\rho}_{0}^{\left[n ; n_{0}\right]}, c f$. Eq. (19b), will in general lie a (constant) few orders of magnitude lower, as it happens, for example, for the data in Fig. 4. Nevertheless, as will be shown in the following, up to an overall global prefactor the discarded weight based on either, $\varepsilon^{\mathrm{D}}$ or $\varepsilon_{\chi}^{\mathrm{D}}$, both behave in an essentially similar fashion. 


\section{B. Application}

The discarded weight $\varepsilon_{(\chi)}^{\mathrm{D}}$ defined in Eq. (21) sensitively depends on the number $M_{\mathrm{K}}$ of states kept or the energy threshold $E_{\mathrm{K}}$. From Fig. 4 one expects a strongly diminishing discarded weight with increasing $M_{\mathrm{K}}$ or $E_{\mathrm{K}}$, a quantitative analysis of which is presented in Figs. 5 and 6 for the SIAM. Figure 5 analyzes the dependence of the discarded weight $\varepsilon_{(\chi)}^{\mathrm{D}}$ on the number $M_{\mathrm{K}}$ of states kept. As seen in panel (a), the discarded weight $\varepsilon_{(\chi)}^{\mathrm{D}}$ strongly decays with $M_{\mathrm{K}}$, with minor variations when a new Wilson shell is fully included without truncation, e.g. at $M_{\mathrm{K}} \in\{256,1024\}$. With panel (a) being a log-log plot, the decay of the discarded weight with $M_{\mathrm{K}}$ rather resembles a polynomial convergence, yet with very large power (on the order of 10). The reason for the slower than exponential decay is due to the strong increase in the density of states $\nu(E)$ of the full many-body eigenspectrum with increasing $E$ as discussed with Figs. 3 and 4.

Together with the analysis of the discarded weight in Fig. 5, an independent physical check for convergence is provided by the numerically computed conductance $g_{0}$ in units of $2 e^{2} / h$ shown in Fig. 5(b). The conductance was calculated via the (spin-resolved) spectral function $A_{(\sigma)}(\omega)=\int \frac{d t}{2 \pi} e^{i \omega t}\left\langle\left\{\hat{d}_{\sigma}(t), \hat{d}_{\sigma}^{\dagger}\right\}\right\rangle_{T}$ of the impurity level, with $g_{0}=\pi \Gamma \int d \omega\left(-\frac{\partial f}{\partial \omega}\right) A(\omega)$. Here the Fermi function $f(\omega)$ and the spectral function $A(\omega)$ are evaluated at small but finite temperature $T \simeq 6 \cdot 10^{-8}$, which is much smaller than the Kondo temperature of $T_{\mathrm{K}} \simeq 1.23 \cdot 10^{-5}$ for given parameter set and corresponds to the energy scale close to the end of the Wilson chain, having $\Lambda=2$ and $N=60$. Expecting $g_{0}=1$ for the symmetric SIAM, the data in Fig. 5 indicates convergence for $M_{\mathrm{K}} \gtrsim 400$. The data for smaller $M_{\mathrm{K}}$ is not yet converged, and therefore (strongly) depends on numerical details, such as nonaveraged z-shifts. 8,9

With $M_{\mathrm{K}}$ being constant, the energy of the topmost kept states can vary significantly with Wilson shell $n$, which directly also leads to a clear dependence of the discarded weight $\varepsilon_{(\chi)}^{\mathrm{D}}$ on $n$. This is shown in the inset to panel (a) for the set of different values of $M_{\mathrm{K}}$ marked in the main panels by the vertical dashed lines. The discarded weight $\varepsilon_{\chi}^{\mathrm{D}}$ clearly varies over more than three orders of magnitude within a single NRG run, irrespective of the actual $M_{\mathrm{K}}$. In particular, one can see that earlier iterations dominate the discarded weight $\varepsilon_{\chi}^{\mathrm{D}}$ for physical reasons. In the strong-coupling regime for $n \gtrsim n_{\mathrm{K}}$ (with iteration $n_{\mathrm{K}} \simeq 35$ corresponding to the energy scale of $T_{\mathrm{K}}$ ), the discarded weight is smallest, while for the intermediate free orbital or local moment regime for $n \lesssim n_{\mathrm{K}}$, these regimes require a larger number of states for comparable numerical accuracy from a physical point of view, indeed.

Given the underlying energy scale separation of the NRG, a straightforward way to obtain a more equally distributed $\varepsilon_{n}^{\mathrm{D}_{(\chi)}}$ is achieved using an energy cutoff $E_{\mathrm{K}}$,
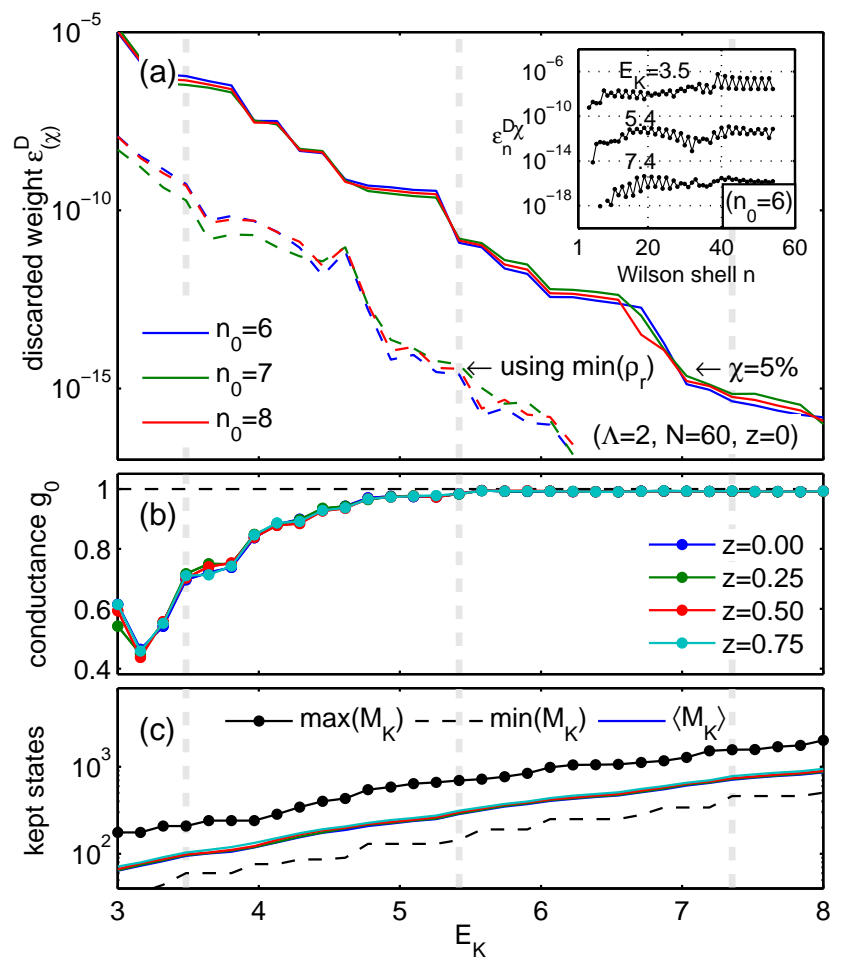

Figure 6: (Color online) Similar analysis as in Fig. 5, yet for truncation with respect to fixed energy $E_{\mathrm{K}}$. For several values of $E_{\mathrm{K}}$ marked by the vertical dashed lines in the main panels, the distribution of the discarded weight $\varepsilon_{n}^{D_{\chi}}$ along the Wilson shell $n$ is shown in the inset to panel (a). With $M_{\mathrm{K}}$ allowed to vary over a wider range, panel (c) shows the correlation of $M_{\mathrm{K}}$ with $E_{\mathrm{K}}$, plotting average, minimum, and maximum of $M_{\mathrm{K}}$ along the Wilson chain. For the average $M_{\mathrm{K}}$, data for different $\mathrm{z}$-shifts is shown (several lines on top of each other, with same color coding as in panel b).

as demonstrated in Fig. 6 for exactly the same system as in Fig. 5 otherwise. For the values of $E_{\mathrm{K}}$ indicated by the vertical dashed lines in the main panels, the inset to Fig. 6(a) shows the distribution of $\varepsilon_{n}^{D_{\chi}}$. By construction, the discarded weight is, up to even-odd oscillations, clearly more uniformly distributed over the Wilson shells as compared to the case of fixed $M_{\mathrm{K}}$ in Fig. 5(a). The discarded weight in panel (a) clearly diminishes exponentially with $E_{\mathrm{K}}$, yet with pronounced intermediate plateaus since the discrete eigenenergies within an NRG run are usually bunched around certain energies. The corresponding average $M_{\mathrm{K}}$ as function of $E_{\mathrm{K}}$, nevertheless, follows a rather smooth monotonic behavior, as shown in panel (c). Given fixed $E_{\mathrm{K}}$, however, clear variations of $M_{\mathrm{K}}$ are seen within a given NRG run, hence also smallest and largest $M_{\mathrm{K}}$ are shown in panel (c). Ignoring iterations without truncation, in given example, typically the largest $M_{\mathrm{K}}$ is required at early iterations, while the smallest $M_{\mathrm{K}}$ are encountered in the strong coupling regime at late iterations $n \gtrsim n_{K}$.

The calculated conductance shown in panel (b) converges clearly more uniformly with increasing $E_{\mathrm{K}}$ as 
compared to Fig. 5(b). In particular, it indicates converged NRG data for $E_{\mathrm{K}} \gtrsim 5.5$, which corresponds to $\varepsilon_{\chi}^{\mathrm{D}} \lesssim 10^{-12}$. Therefore in both settings, for constant $M_{\mathrm{K}}$ in Fig. 5 as well as for constant $E_{\mathrm{K}}$ in Fig. 6, convergence of the physical data is found for a similar discarded weight of $\varepsilon_{\chi}^{\mathrm{D}} \lesssim 10^{-12}$ with a negligible dependence on $n_{0}$. This value therefore is considered a sufficient bound in accuracy to capture the main physics, with other quantities such as the NRG energy flow diagram already also well converged.

Alternatively, using the plain minimum of the eigenvalues of the reduced density matrices in Eq. (19b), this leads to convergence for $\varepsilon^{\mathrm{D}} \lesssim 10^{-16}$. Given that $\varepsilon^{\mathrm{D}}$ refers to the minimum eigenvalue in the kept space, $\varepsilon^{\mathrm{D}}$ consistently lies about three orders of magnitudes lower than $\varepsilon_{\chi}^{\mathrm{D}}$ and is considered a lower bound to the actual discarded weight. While $\varepsilon^{\mathrm{D}}$ fluctuates slightly more strongly compared to $\varepsilon_{\chi}^{\mathrm{D}}$ owing to the fact that it is not an averaged quantity such as $\varepsilon_{\chi}^{\mathrm{D}}$, it nevertheless follows a similar consistent picture in terms of convergence with the number $M_{\mathrm{K}}$ of states kept or the energy $E_{\mathrm{K}}$ used for truncation. In this sense, either discarded weight, $\varepsilon^{\mathrm{D}}$ as well as $\varepsilon_{\chi}^{\mathrm{D}}$, can be used quite generally as a quantitative measure, indeed, to demonstrate accuracy within the NRG. In order to avoid confusion, however, it shall be made clear which one is used.

\section{ENTANGLEMENT SPECTRA}

The reduced density matrices $\hat{\rho}_{0}^{\left[n ; n_{0}\right]}$ clearly also carry physical information in terms of entanglement along the Wilson chain. This is provided by the high end of their spectral decomposition. There the exact details of the largest eigenvalues of $\hat{\rho}_{0}^{\left[n ; n_{0}\right]}$ are of interest, which do vary with $n_{0}$ over a wider range depending on the underlying physics. Hence, in the following, the actual entanglement spectra will be calculated with respect to the reduced density matrices $\hat{\rho}_{0}^{[n]}$ of the overall ground state of the system,

$$
\hat{\rho}_{0}^{[n]} \equiv \lim _{n_{0} \rightarrow \infty} \hat{\rho}_{0}^{\left[n ; n_{0}\right]} \simeq \hat{\rho}_{0}^{[n ; N-n]} .
$$

The length $N$ of the Wilson chain is taken sufficiently large, such that the energy scale of the last iteration $N$ is much smaller than any other energy scale in the system. Temperature is therefore essentially zero. For comparison, also the truncated entanglement spectra will be calculated from $\hat{\rho}_{0}^{\left[n ; n_{0}\right]}$ for finite small $n_{0}$, with $n_{0}$ specified in context. Motivated by the discussion following Eq. (6), the latter analysis can be linked to finite temperature settings.

\section{General definition}

The partitioning of the Wilson chain into two parts, the chain up to and including site $n$ (part A), and the traced
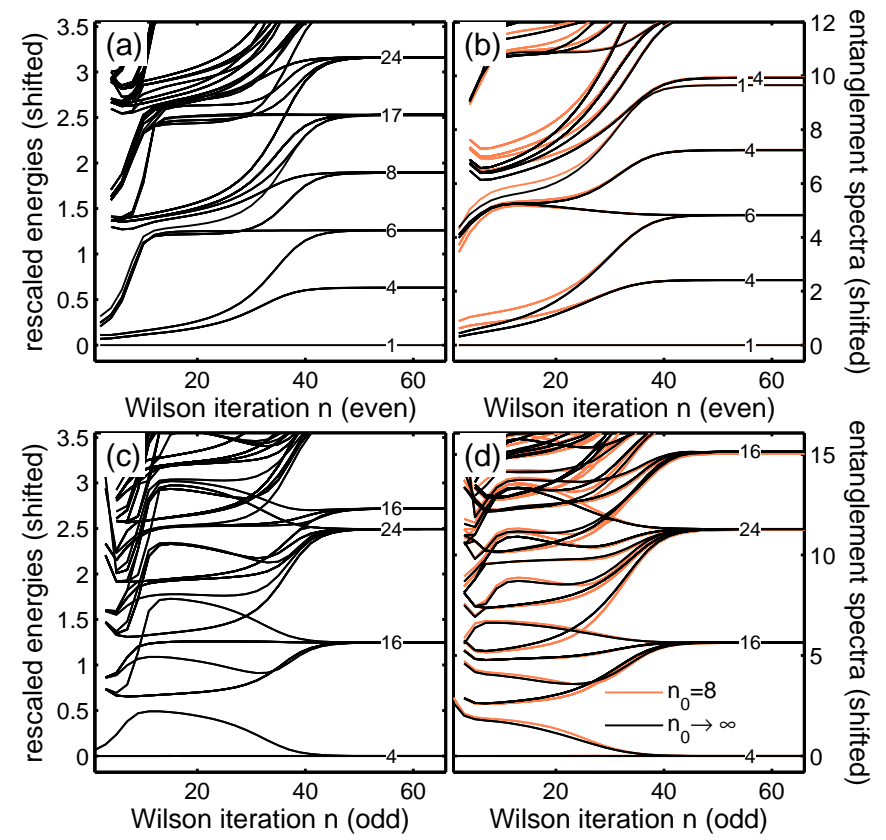

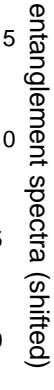

Figure 7: (Color online) Comparison of the standard NRG energy flow diagram (left panels) to the entanglement flow diagram (right panels) for the symmetric SIAM $[U=0.2$, $\varepsilon_{d}=-U / 2, \Gamma=0.01, T_{\mathrm{K}}=1.2 \cdot 10^{-5} ; \Lambda=2, M_{\mathrm{K}}=512$, $N=80]$, with top (bottom) panels for even (odd) iterations, respectively. In addition to the actual entanglement flow diagram obtained from the ground state of the last iteration at $N=80$ (black lines), also the truncated entanglement flow diagram is shown, using $n_{0}=8$ (orange (gray) lines). For better comparison with the energy flow diagram, the entanglement spectra (right panels) are also shifted at every iteration with respect to the smallest entanglement energy $\min (\xi)$. The yscale of the entanglement spectra was adjusted to best match the energy fixed point spectrum in the left panels. Degeneracies of energies at large $n$, i.e. lines lying indistinguishably on top of each other, are specified by the numbers on top of the lines in all panels.
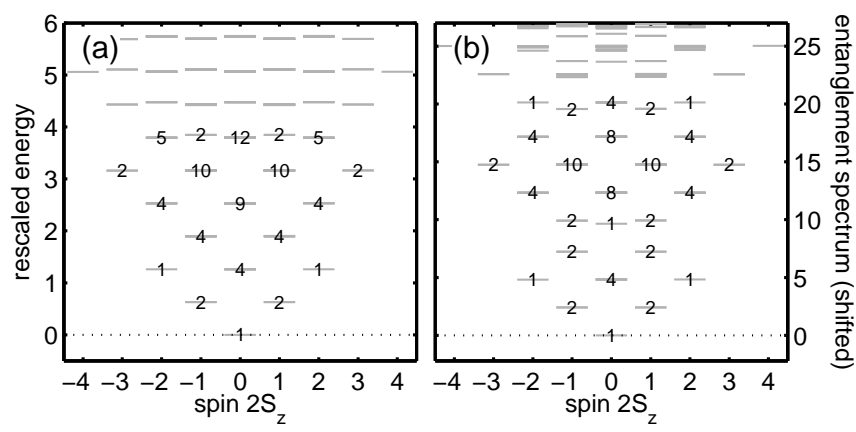

Figure 8: (Color online) Comparison of spin-resolved fixed point spectra for the symmetric SIAM in Fig. 7 in the SC regime $(n=60)$. Panel $(\mathrm{a})[\mathrm{b}]$ show the energy [entanglement] fixed point spectrum, respectively, vs. spin symmetry quantum number $S_{z}$. For all low-energy multiplets the underlying (approximate) degeneracy is indicated. The entanglement spectrum is shifted w.r.t. to its lowest energy and scaled to

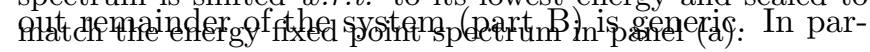


ticular, this allows to make use of the recently introduced entanglement spectra (ES) ${ }^{19}$ for the physical characterization of a given wave function. Here these entanglement spectra provide a powerful tool for the systematic analysis of the physical correlations in the reduced density matrices $\hat{\rho}_{0}^{[n]}$ in Eq. (22).

Consider a given wave function of a some system partitioned into parts $\mathrm{A}$ and $\mathrm{B}$. The reduced density matrix $\hat{\rho}_{A} \equiv \operatorname{tr}_{B}(\rho)$ is obtained by tracing out part $B$ of the overall density matrix $\rho$. Within this setting, the entanglement spectrum is defined as the spectrum of the fictitious Hamiltonian $\hat{H}_{\rho}^{A}, 19$

$$
\hat{\rho}_{A}=: \exp \left(-\hat{H}_{\rho}^{A}\right)
$$

One may assume an effective inverse temperature $\beta:=1$ in order to make contact with a thermal density matrix. This $\beta$ also sets the (otherwise arbitrary) energy scale in the per se dimensionless $\hat{H}_{\rho}^{A}$. With $\hat{\rho}_{A}$ a positive operator, the entanglement spectrum $\xi_{r}$ is defined as the eigenvalues of $\hat{H}_{\rho}^{A}$, i.e.

$$
\xi_{r}:=-\log \rho_{r}
$$

with $\rho_{r}$ the spectral decomposition of the reduced density matrix $\hat{\rho}_{A}$. Particular information can be read off from the entanglement spectrum as soon as there is a rich amount of quantum numbers specifying the entanglement levels and when entanglement gaps appear which separate a low-lying generic set of levels from irrelevant background correlations. ${ }^{19-21}$ The spectra $\rho_{r}$ and $\xi_{r}$ are independent of whether A or B is traced out, while of course, they are dependent on the specific choice of the partitioning. For entanglement spectra, the partitioning typically occurs in real space for gapped systems, analyzing the edge of the thus created boundary, while for gapless systems momentum space is preferred. ${ }^{20}$ The second case then is consistent with the systematic NRG prescription of energy scales based on the underlying discretization in energy (momentum) space.

By construction, the dominant correlations between systems $A$ and $B$ correspond to the lowest entanglement energies $\xi_{r}$, while weaker correlations will rise to higher energies. By tracing out a major part of the system, entanglement spectra provide significantly more information, say, than just the entanglement entropy between $A$ and $B$. In particular, it has been shown that it provides finger prints of the underlying physics, and as such allows to characterize the physical nature of a given wave function. ${ }^{19,20}$ This analysis is therefore entirely targeted at a given (ground state) wave function, without any further reference to an underlying physical Hamiltonian that it may have originated from.

\section{Application to NRG}

The general concept of the entanglement spectra can be readily transferred to the NRG. At each iteration $n$, the reduced density matrix $\hat{\rho}_{0}^{[n]}$ in Eq. (22) is computed and diagonalized, with its eigenspectrum mapped onto the entanglement spectrum in Eq. (23). Collecting these spectra and plotting them vs. iteration index $n$ for even and odd iterations separately, the result will be referred to as entanglement flow diagram, in complete analogy to the standard energy flow diagrams of the NRG. For comparison, also the truncated entanglement spectra for finite small $n_{0}$ will be analyzed, which in their combination will be referred to as truncated entanglement flow diagram. In either case, the entanglement spectra are obtained in a backward sweep, purely based on the iterative low-energy Hilbert-space decomposition of a prior NRG run in terms of the A-tensors in Eq. (3). This is in contrast to the energy flow diagram, which is calculated with increasing shell index $n$ in a forward sweep making explicit reference to the Hamiltonian.

The entanglement spectra were calculated for the symmetric SIAM in the absence of magnetic field. The resulting entanglement flow diagram is presented in Fig. 7 together with a direct comparison to the standard NRG energy flow diagram. The data is plotted for even (odd) Wilson shells $n$ in the upper (lower) panels, respectively. The energy flow diagram, shown in the left panels, clearly distinguish the well-known physical regimes of the SIAM, namely the free orbital regime $(\mathrm{FO} ; n \lesssim 10$ ), the local moment regime $\left(\mathrm{LM} ; 10 \lesssim n \lesssim n_{\mathrm{K}}\right)$, and the strong coupling regime $\left(\mathrm{SC} ; n \gtrsim n_{\mathrm{K}}\right)$, where $n_{\mathrm{K}} \simeq 35$ corresponds to the energy scale of the Kondo temperature $T_{\mathrm{K}}=1.2 \cdot 10^{-5}$, having $\Lambda=2$. All degeneracies for $n>n_{\mathrm{K}}$ are explicitly specified in Fig. 7. In particular, for even iterations, the ground state is unique throughout, e.g. the Kondo singlet for $n>n_{\mathrm{K}}$ (panel a), while for odd-iterations the ground state space at small energies is four-fold degenerate due to the particle-hole symmetric parameter set (panel c).

Interestingly, a very similar picture emerges from the entanglement flow diagram in right panels, Fig. $7(\mathrm{~b}+\mathrm{d})$ (black lines). For comparison, also the truncated entanglement spectra are shown using $n_{0}=8$ (orange (gray) lines), which in given case converge rapidly, in fact exponentially, with increasing $n_{0} \lesssim 10$ towards the actual entanglement flow diagram. The latter then mimic the energy flow diagram in the left panels over a wide range. For example, the convergence towards the Kondo fixed point occurs around similar iterations, and even the degeneracies of the lowest states of the energy flow diagram are exactly recovered by the entanglement spectra. The latter also holds on the symmetry-resolved level, as demonstrated in Fig. 8 for the even iteration $n=60$ (see later discussion). Nevertheless, looking more closely, a few notable qualitative differences of the entanglement flow diagrams in right panels of Fig. 7 are seen compared to the energy flow diagrams in the left panels. Overall, the entanglement flow diagrams appear shifted by about 5 iterations to larger energies. This can be understood, considering that the entanglement spectra are calculated for enlarged systems together with the rapid convergence 


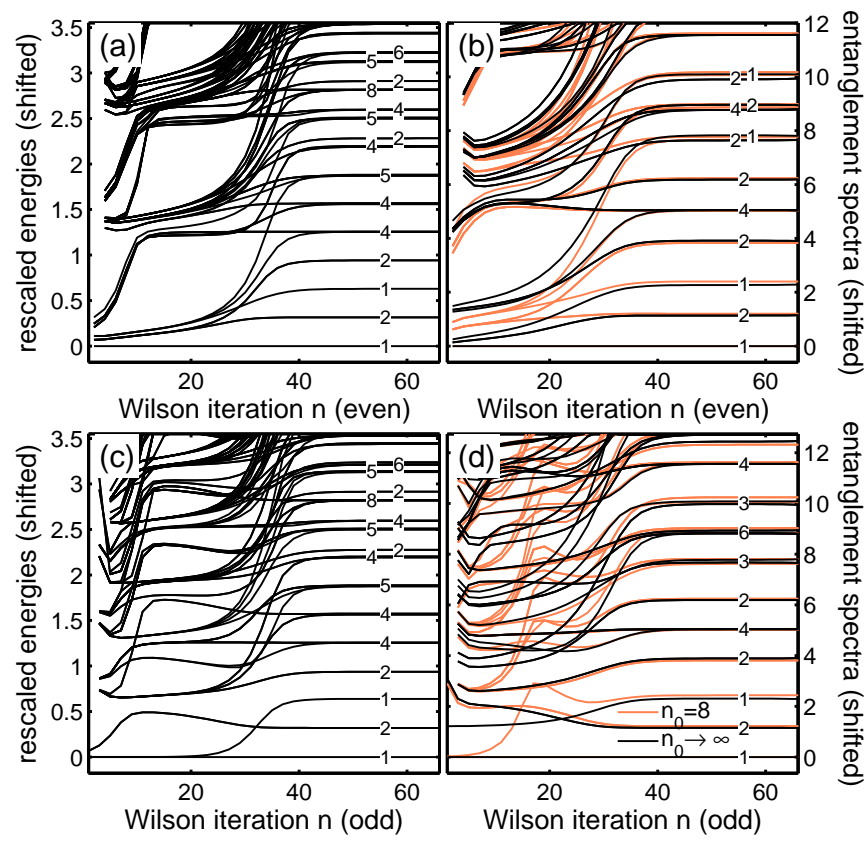

Figure 9: (Color online) Comparison of the standard NRG energy flow diagram (left panels) to the entanglement flow diagram (right panels) for the SIAM at finite magnetic field (same analysis as in Fig. 7, otherwise, see caption there for details, with same model parameters, except $B=2 \cdot 10^{-5} \simeq$ $\left.1.6 T_{\mathrm{K}}\right)$.

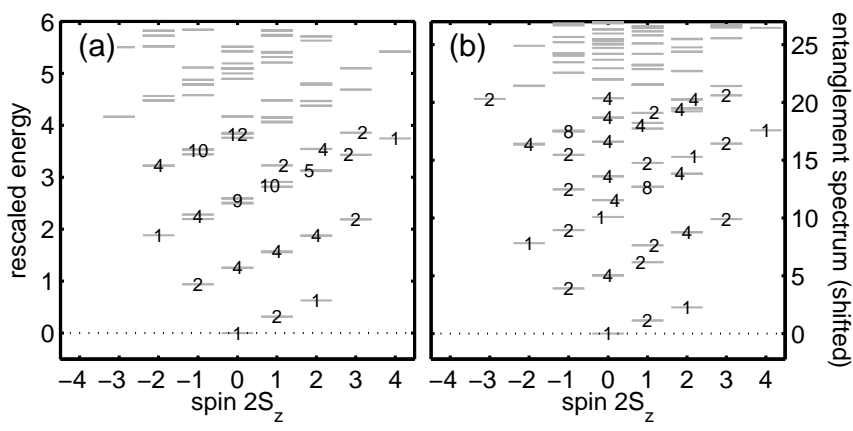

Figure 10: (Color online) Comparison of spin-resolved fixed point spectra for the SIAM at finite magnetic field in Fig. 9 at the even iteration $n=60$ (similar analysis as in Fig. 8 otherwise).

with increasing $n_{0}$ in given case. However, there are further pronounced differences with the energy flow diagram for the earliest iterations in the FO regime, $n \lesssim 10$.

These latter differences in the entanglement flow diagram can be significantly enhanced by turning on a magnetic field on the order of the Kondo temperature, as shown in Fig. 9 for $B=1.6 T_{\mathrm{K}}$. This corresponds to the energy scale at iteration $n_{\mathrm{B}} \simeq 32$, given $\Lambda=2$. The magnetic field has been chosen such, that for late iterations $n \gg n_{\mathrm{B}}$ the fixed point spectrum for even and odd iterations become essentially the same (compare the low-energy fixed point spectra in panels a(b) to c(d), respectively). Due to the magnetic field, the Kondo singlet (previously the unique state at even iterations) is largely destroyed for $n \gtrsim n_{\mathrm{B}}$ with a sizeable magnetization at the impurity. Clearly, the NRG eigenbasis at early iterations $n<n_{\mathrm{B}}$ does not yet know about the small energy physics to come (e.g. the small $B \sim T_{\mathrm{K}}$ applied in given case). Therefore the energy flow diagram essentially remains unaltered there, when compared to the case without magnetic field in Fig. $7(\mathrm{a}+\mathrm{c})$. The flow changes strongly only starting from the energy scale of the magnetic field value, i.e. for $n>n_{\mathrm{B}}$ where it moves into a different fixed-point spectrum. In particular, there also emerges a unique state now in the energy flow diagram for odd iterations for $n \gg n_{\mathrm{B}}$, i.e. the symmetry broken spinful state favored by the magnetic field. By including magnetic field, the entanglement flow diagram shows pronounced differences from the energy flow diagram for $n \lesssim n_{\mathrm{B}}$, which includes large portions of the LM regime. While the energy spectrum up to and including site $n$ is ignorant of the low-energy physics to come, this very low-energy physics is captured by the reduced density matrices and thus reflected in the entanglement flow diagram. $^{24}$

Consider the entanglement spectra derived from the overall ground state (black lines) in Fig. $9(\mathrm{~b}+\mathrm{d})$. In panel (d) the ground state remains unique throughout, i.e. remembers the symmetry broken magnetic state, determined at much lower energy scales, all the way up to the largest energies. Within the split-up lowest energy space with subsequent degeneracies [1-2-1] in panel (d) for $n \gg n_{\mathrm{B}}$ (to be called [1-2-1] configuration), the first and second excited states cross each other with decreasing $n$ leading to a [1-1-2] configuration for small $n$, i.e. large energies. Nevertheless, the singly degenerate excited state clearly remains split-off, and does not merge with the ground state, which is in strong contrast to the energy flow diagram in panel (c) with a [2-2] configuration for $n \ll n_{\mathrm{B}}$. This degeneracy in the ground state space that is ignorant of the small magnetic field is partly reflected only in the truncated entanglement flow diagram. Using small $n_{0}$ (orange (gray) lines in panels d), this eventually also misses the low energy physics. Therefore these spectra in panel (d) eventually are also in a [2-2] configuration for the smallest $n$, with a more irregular transient behavior with increasing $n$. A similar trend is also observed for even iterations in panels $(a+b)$. While the ground state remains unique for all iterations in both panels, the entanglement flow in panel (b) tends to split off the excited levels right above the lowest [1-2-1] state space configuration for small $n$. For the truncated entanglement flow, on the other hand, the lines of these excited levels remain entangled with higher excitations, which is similar to the situation in the energy flow diagram in panel (a).

Nevertheless, the low-energy fixed-point spectra for $n \gg n_{\mathrm{B}}$ again agree well for both the energy and entanglement flow diagram in Fig. 9, which again also holds 
for the symmetry-resolved spectra, as demonstrated for the even iteration $n=60$ in Fig. 10. This agreement in the spectra of the stable low-energy fixed point, present in both the non-magnetic as well as the magnetic case, is understood as a generic feature. There both, the energy eigenstates as well as the reduced density matrices are deeply rooted in the low-energy physics, i.e. of the overall ground state of the system at $T \rightarrow 0$, and hence present a consistent description of the system.

The detailed structure of the energy fixed point spectra provides clear physical information. ${ }^{1,2}$ This includes, for example, phase shifts if a Fermi-liquid point of view is supported as is the case for the SIAM. This then directly explains all of the splittings and degeneracies in the low energy sector of the energy fixed point spectra. For example, consider the energy spectrum in Fig. 8(a) for the fully symmetric SIAM in the non-magnetic case. Note that while spin-resolved spectra are shown in Fig. 8, in given case the charge-resolved spectra would look exactly the same due to particle-hole symmetry. With the spectra shown for an even iteration, the ground state is unique, i.e. represents the Kondo singlet with $S_{z}=0$. The first excited states for $S_{z}=+\frac{1}{2}$, correspond to an extra particle with spin-up or a hole with spin-down. Given particle-hole symmetry, both processes have the same energy $\delta / 2=0.63$ (in rescaled energy units), and hence are two-fold degenerate, indicated by the number on top of the level in Fig. 8. By symmetry, the same excitations exist for $2 S_{z}=-1$, leading to the [2-2] degeneracy (4 states) in the lowest excitations in Fig. 8(a). The next higher excitation combines two of above processes. This leads to a total of 6 excitations, all with energy $\delta$ and distributed over $2 S_{z} \in\{-2,0,+2\}$. Here two of the excitations at $2 S_{z}=0$ correspond to the extraction or annihilation of two particles with opposite spin. This fully explains the [1-4-1] degeneracy of the excited states at energy $\delta=1.26$ in Fig. 8(a), and also the combined 6fold degeneracy seen in the energy flow diagram seen at this energy in Fig. 7(a). The argument can be continued along similar lines to explain the [4-4] (8 states) and [49-4] (17 states) degenerate subspaces of the next higher excitations. Excitations with even higher energy eventually have missing levels due to NRG truncation.

The same analysis as for the energy spectra, however, cannot be applied with equal rigor to the entanglement spectra. While the ground state [1] and the lowest [2-2] and [1-4-1] excitations in Fig. 8(b) fully agree in symmetries, degeneracy and also in the precise relative level spacing, the next higher [4-4] excitation in panel (a) is broken up in Fig. 8(b), with some of the levels shifting to higher entanglement energy. Nevertheless, the degenerate set [2-10-10-2] further up in energy still again equally appears for both, energy and entanglement spectra.

The same analysis as in Fig. 8, is repeated for the magnetic case in Fig. 10 for the same even iteration $n=60$. Despite the rather different level spectrum for large $n$ in the flow diagram in Fig. 9, the actual spin-resolved fixed point spectrum is qualitatively very similar to the non- magnetic case in Fig. 8. Aside an overall tilt of the level structure, all degeneracies and level positions of the lower part of the energy spectrum in panel (a) are again fully described by elementary single-particle excitations. The underlying reason for this similarity of the fixed points spectra in the magnetic and non-magnetic case is that, apart from the (screened) impurity spin, the system is well described by an effective Fermi-liquid picture. With the low energy fixed point spectra well reflected in the entanglement spectra, a similar tilt in the level structure is also observed in Fig. 10(b) when compared to Fig. 8(b). Note, for example, that to the lower left of the spectrum the same [1-2-1], as well as the [2-4-2, 2-4-2] state sequence with increasing energy is seen.

\section{SUMMARY AND OUTLOOK}

The reduced density matrices of the NRG by tracing out the low-energy sector have been analyzed in detail. The low end of their eigenspectra was used to estimate the discarded weight $\varepsilon_{(\chi)}^{\mathrm{D}}$ in Eqs. (19-21) as a quantitative and site-resolved measure of the accuracy within the NRG. While, in principle, the same reduced density matrices could also be utilized as the basis for an altered truncation criteria similar to the DMRG, this, however, requires sufficiently large $M_{\mathrm{K}}$ to start with. In practice, this is sufficiently close to a truncation with respect to an energy cutoff $E_{\mathrm{K}}$. Either way, all of this can be easily and quickly checked using the proposed analysis in terms of the discarded weight which provides a useful quantitative tool.

Furthermore, the dominant correlations of the reduced density matrices were analyzed in terms of their entanglement spectra. Due to the NRG flow towards small energy scales, these spectra can be combined into entanglement flow diagrams. There different physical regimes can be identified similar to the standard NRG energy flow diagrams. Considering that the entanglement spectra are obtained solely based on the wave function, the agreement of the low-energy fixed point spectra are stunning. A possible larger disagreement at higher energies, i.e. for earlier Wilson shells, on the other hand, depends on the specific physical situation. Given the NRG background, as an outlook this appears to suggest the following. For all energy shells (iterations) $n$ where the entanglement spectrum is quantitatively comparable to the NRG energy spectrum for the lowest set of states, the reduced density matrices themselves are not crucially important in the description of the system. Instead, they may be replaced by thermal density matrices in the NRG eigenbasis. In a sense, by tracing out the low-energy sector, the resulting reduced density matrices maintain an approximate thermal character, with implications to thermalization at a given energy shell. ${ }^{23}$ For energy shells with a qualitative difference between the energy and entanglement spectra, however, the reduced density matrices are crucially important to capture the correct physics in 
the NRG calculation that explicitly uses data from such energy shells.

A detailed analysis of the deeper connection and the explicit differences between the energy and the entanglement spectra appears interesting, yet is out of the scope of this paper. In particular, it also appears instructive to analyze the entanglement spectra for non-fermi liquid systems such as the symmetric two-channel Kondo model, as the analysis presented in this paper suggests a strong physical connection of the entanglement spectra to the underlying physics.

\section{Acknowledgments}

I want to thank Jan von Delft for a critical review of the script, and also Ronny Thomale for helpful comments on entanglement spectra. This work has received support from the German science foundation (DFG: TR-12, SFB631, NIM, and WE4819/1-1).

1 Kenneth G. Wilson. Rev. Mod. Phys., 47 (4), 773-840, 1975.

2 Ralf Bulla, Theo Costi, and Thomas Pruschke. Rev. Mod. Phys., 80, 395, 2008.

3 Steven R. White. Phys. Rev. Lett., 69 (19), 2863-2866, 1992.

${ }^{4}$ U. Schollwöck. Rev. Mod. Phys., 77 (1), 259-315, 2005.

5 Ulrich Schollwöck. Ann. Phys., 326, 96-192, 2011.

${ }^{6}$ H. R. Krishna-murthy, J. W. Wilkins, and K. G. Wilson. Phys. Rev. B, 21 (3), 1003-1043, 1980.

7 M. Yoshida, M. A. Whitaker, and L. N. Oliveira. Phys. Rev. B, 41, 9403, 1990.

8 L. N. Oliveira. Braz. J. Phys, 22, 155, 1992.

${ }^{9}$ Rok Žitko and Thomas Pruschke. Phys. Rev. B, 79 (8), 085106, 2009.

10 James Demmel, Jack Dongarra, Axel Ruhe, and Henk van der Vorst. Templates for the solution of algebraic eigenvalue problems: a practical guide. Society for Industrial and Applied Mathematics, Philadelphia, PA, USA, 2000. ISBN 0-89871-471-0.

11 F. B. Anders and A. Schiller. Phys. Rev. Lett., 95, 196801, 2005.

12 Andreas Weichselbaum and Jan von Delft. Phys. Rev. Lett., 99 (7), 076402, 2007.

13 A. Weichselbaum, F. Verstraete, U. Schollwock, J. I. Cirac, and Jan von Delft. Phys. Rev. B, 80 (16), 165117, 2009.

14 Stefan Rommer and Stellan Östlund. Phys. Rev. B, 55 , 2164, 1997.

15 Robert Peters, Thomas Pruschke, and Frithjof B. Anders. Phys. Rev. B, 74 (24), 245114, 2006.

16 Walter Hofstetter. Phys. Rev. Lett., 85 (7), 1508-1511, 2000.

17 A. I. Tóth, C. P. Moca, Ö. Legeza, and G. Zaránd. Phys.

Rev. B, $78(24), 245109,2008$

18 Hamed Saberi, Andreas Weichselbaum, and Jan von Delft. Phys. Rev. B, $\mathbf{7 8}$ (3), 035124, 2008.

19 Hui Li and F. D. M. Haldane. Phys. Rev. Lett., 101 (1), 010504, 2008.

20 R. Thomale, D. P. Arovas, and B. Andrei Bernevig. Phys. Rev. Lett., 105 (11), 116805, 2010.

21 R. Thomale, A. Sterdyniak, N. Regnault, and B. Andrei Bernevig. Phys. Rev. Lett., 104 (18), 180502, 2010.

22 T. A. Costi. Phys. Rev. B, 55 (5), 3003-3009, 1997.

23 D. Poilblanc. Phys. Rev. B, 84, 045120, 2011.

24 Note that it was exactly this kind of reasoning, for example, that led to the success of density-matrix-based NRG methods for dynamical properties, starting with DMNRG. ${ }^{16}$ For the SIAM, for example, the reduced density matrices, while not crucially important in the absence of magnetic field, are absolutely essential for the correct description of spectral correlations at finite magnetic field. There it is intuitively clear that the spin-resolved spectral function $A_{\sigma}(\omega)$ at the impurity at small temperatures $T \ll T_{\mathrm{K}}$ in the presence of a magnetic field $B>T_{\mathrm{K}}$ redistributes significant spectral weight at large energies $|\omega| \gg T_{\mathrm{K}}$, which accounts for the breakup of the Kondo singlet. Within the NRG, this thus translates into a feedback from small to large energies, which is captured correctly only after including the reduced density matrices for the remainder of the system. ${ }^{16}$ Yet contributions from all NRG shells are required to cover the full spectral range of dynamical correlation functions. ${ }^{22}$ A clean combined prescription for this was finally provided by the FDM-NRG approach $^{12}$ based on complete basis sets. ${ }^{11,15}$ 